This item was submitted to Loughborough's Research Repository by the author.

Items in Figshare are protected by copyright, with all rights reserved, unless otherwise indicated.

\title{
In-process surface profile assessment of rotary machined timber using a dynamic photometric stereo technique
}

PLEASE CITE THE PUBLISHED VERSION

http://dx.doi.org/10.1177/0959651811433121

PUBLISHER

Sage Publications / @ IMechE

VERSION

AM (Accepted Manuscript)

LICENCE

CC BY-NC-ND 4.0

\section{REPOSITORY RECORD}

Ogun, Philips S., Michael R. Jackson, and Robert M. Parkin. 2019. "In-process Surface Profile Assessment of Rotary Machined Timber Using a Dynamic Photometric Stereo Technique”. figshare.

https://hdl.handle.net/2134/15084. 
This item was submitted to Loughborough's Institutional Repository (https://dspace.lboro.ac.uk/) by the author and is made available under the following Creative Commons Licence conditions.

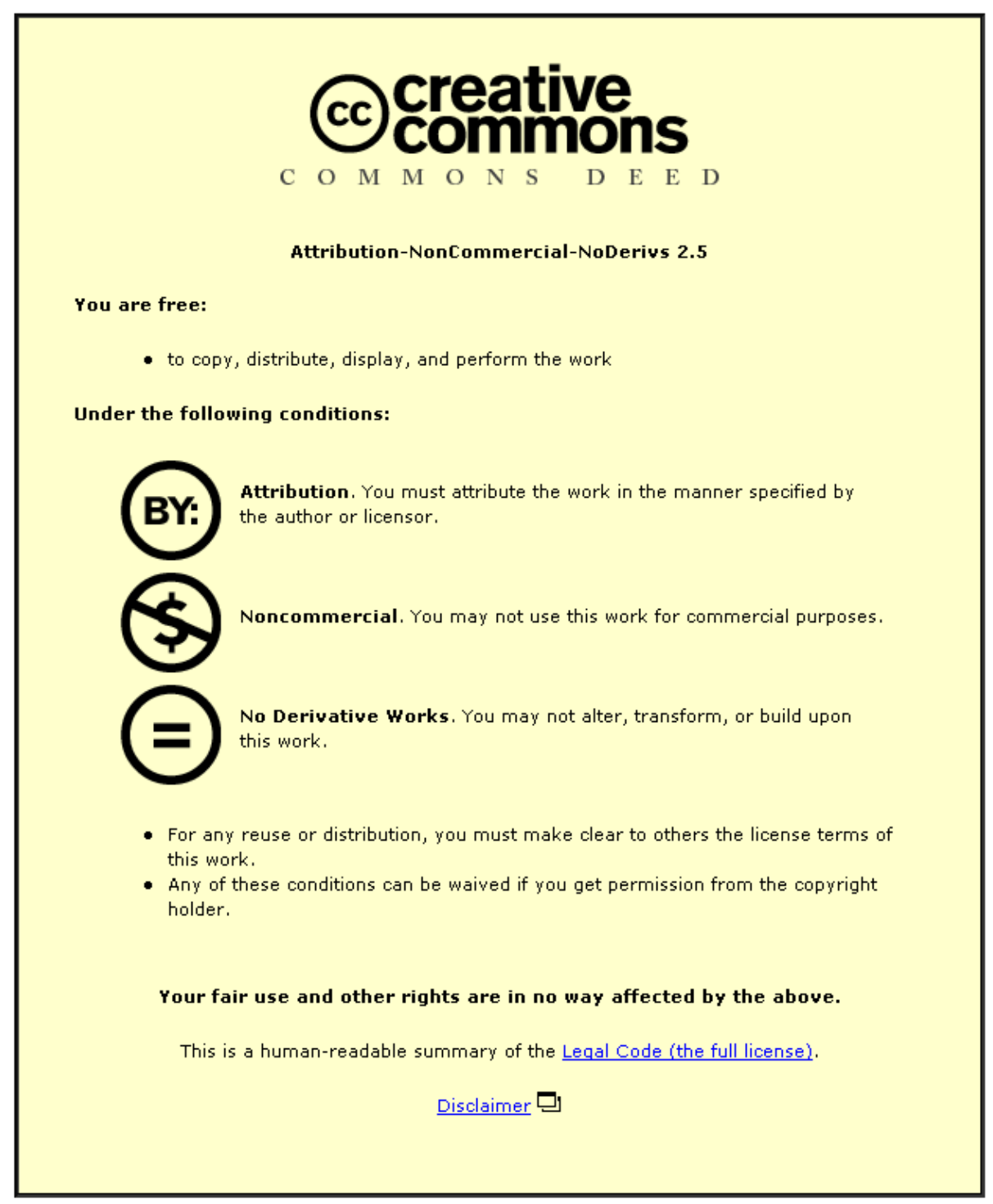

For the full text of this licence, please go to: http://creativecommons.org/licenses/by-nc-nd/2.5/ 


\title{
In-process Surface Profile Assessment of Rotary Machined Timber Using Dynamic Photometric Stereo Technique
}

\author{
Philips S. Ogun, Michael R. Jackson, Robert M. Parkin \\ Mechatronics Research Centre, Wolfson School of Mechanical and Manufacturing \\ Engineering, Loughborough University, Loughborough, UK
}

\begin{abstract}
Machining operations have advanced in speed and there is an increasing demand for higher quality surface finish. It is therefore necessary to develop real-time surface inspection techniques, which will provide sensory information for controlling the machining processes. This paper describes a practical method for real-time analysis of planed wood using the photometric stereo (PS) technique. Earlier research revealed that the technique is very effective in assessing surface waviness on static wood samples. In this paper, the PS method is extended to real industrial applications where samples are subjected to rapid movements. Surface profiles extracted from the dynamic PS method are compared with those from the static measurements and the results show that there is a high correlation between the two methods.
\end{abstract}

Keywords: Wood machining, surface waviness, surface inspection, dynamic photometric stereo 


\section{$1 \quad$ Introduction}

Rotary machining of timber is similar to the milling of metals. During the process, the timber workpiece is fed into a revolving cutterhead. The cutters remove wood chips from the surface of the workpiece, thereby creating series of waves known as cuttermarks on the workpiece [1]. The sizes and uniformity of the cuttermarks are used to determine the quality of the surface finish. As quality control demands increase, there is the need to perform in-process assessment of the cuttermarks so that necessary control actions can be made when the surface quality deviates from the desired.

Research into surface profile inspection has received lesser attention in the woodworking industry compared to the metalworking industry. The popular contactbased measurement using Stylus profilometer has been found to be unsuitable for inprocess surface profile assessment because of its limited speed. Analysis of some of the currently developed non-contact wood surface profile measurement techniques based on machine vision can be found in [2].

An image processing system was developed by Hesselbach et al. [3] for measuring the cuttermarks on a planed wood. The processing algorithm is based on finding the average intensity values in each row of the images. The method is suitable for assessing moving wood samples at high feed rate because of its simplicity. However, it is unable to reveal any information about the heights of the cuttermarks. The light sectioning method [4] and the photometric stereo technique $[5,6]$ have also been used to assess the waviness on static wood samples. 
The fundamental of this paper has been reported in $[5,6]$ where a two-image PS technique is used to assess the surface waviness of static wood samples. In principle, the conventional PS (CPS) is only suitable for analysing static surfaces because it requires that the position of the object remains fixed with respect to the camera during the acquisition of the images. The so-called correspondence problem now arises if the sample is subjected to some movements during the acquisition of the successive image pairs. The main aim of this paper is to improve on the work done in $[5,6]$ by extending the PS method to the assessment of cuttermarks on dynamic wood samples.

Some existing applications have already applied the PS technique to moving surface analysis [7 - 9]. This is termed as dynamic photometric stereo (DPS). A case study on the online inspection of ceramic tiles is presented in [8]. A method known as spatial multiplexing is used to achieve a synchronised capture of multiple images. The registration of the images is achieved through pre-calibration of the sample speed. The limitation of this method is that it can only work for a predetermined speed and the image capture synchronisation must be fixed. As such, the relative movement between the imaging system and the object needs to be precisely controlled. There are other applications of DPS where single coloured images are utilised instead of multiple graylevel images [10].

\section{Theory of Photometric Stereo}

Although the detailed idea behind the use of the PS technique to extract cuttermarks from planed wood has been reported in [5,6], the basic theory is still presented here for completeness. The PS technique is used to estimate local gradient of a surface from 
multiple images captured in a fixed spatial location but under different illumination directions [11-13]. The fundamental theory that underpins the application of the PS technique to wood surfaces is the assumption that wood exhibits Lambertian (diffuse) reflectance. Although total reflection from polished wood usually comprises of both specular and diffuse reflection, unpolished wood exhibits nearly diffuse reflectance [14]. The geometry of the Lambertian surface imaging system is shown in Fig. 1.

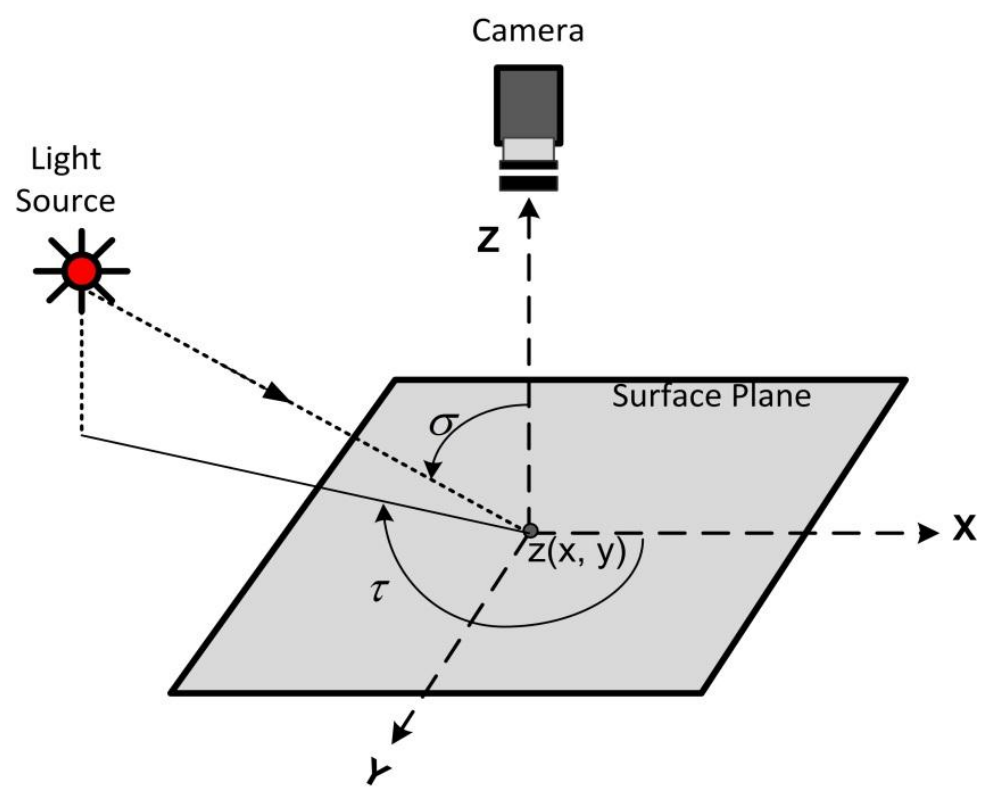

Fig. 1 Geometry of the Lambertian surface imaging system [5]

where

- The $X Y$ plane is coincident with the object surface and the $Z$ axis is perpendicular to the object surface

- The $Z$ axis is aligned with the viewing direction

- $\tau$ is known as the azimuth or tilt angle of the light source. It is the angle between the $X$ axis and the light path, measured in the $X Y$ plane 
- $\sigma$ is known as the zenith or slant angle of the light source. It is the angle between the light path and the camera direction.

According to Lambert's law, the reflection from a point $(x, y)$ on a Lambertian surface is given by [14]

$$
I(x, y)=I_{o} \rho(x, y)\left(\frac{-p(x, y) \cos \tau \sin \sigma-q(x, y) \sin \tau \sin \sigma+\cos \sigma}{\sqrt{\left(p^{2}(x, y)+q^{2}(x, y)+1\right)}}\right)
$$

where

- $I(x, y)$ is the intensity reflected at the point $(x, y)$

- $I_{o}$ is the intensity of the incident light

- $\rho(x, y)$ is the point albedo; it is the fraction of incident light that is being reflected from the point $(x, y)$

- $\quad p$ and $q$ define the gradient of the surface at any local point in the $x$ and $y$ directions respectively.

\subsection{Surface Profile Recovery using PS}

Ideally, a rotary planed wood surface does not have cuttermarks height variation along the $Y$ axis, i.e. $q(x, y) \cong 0$. For this reason, the three-dimensional surface topography can be represented by a two-dimensional surface profile $[5,6]$. Eq. 1 then reduces to the following equation:

$$
I(x)=I_{o} \rho(x)\left(\frac{-p(x) \cos \tau \sin \sigma+\cos \sigma}{\sqrt{\left(p^{2}(x)+1\right)}}\right)
$$


If an object is illuminated from two distinct locations, the surface reflectance will be the same but the reflectance map will be different $[15,16]$. Suppose that two lights having approximately equal intensities $I_{o}$, are located at the same zenith angle $\sigma$, but at different azimuth angles $\tau_{1}=0^{\circ}$ and $\tau_{2}=180$ as shown in Fig 2 , then the following equations can be obtained from Eq. 2:

$$
\begin{aligned}
& I_{1}(x)=I_{o} \rho(x)\left(\frac{-p(x) \sin \sigma+\cos \sigma}{\sqrt{\left(p^{2}(x)+1\right)}}\right) \\
& I_{2}(x)=I_{o} \rho(x)\left(\frac{-p(x) \sin \sigma+\cos \sigma}{\sqrt{\left(p^{2}(x)+1\right)}}\right)
\end{aligned}
$$

Dividing Eq. 3a by Eq.3b gives

$$
p(x)=\frac{I_{2}(x)-I_{1}(x)}{I_{2}(x) \cos \tau_{1}-I_{1}(x) \cos \tau_{2}} \cdot \frac{1}{\tan \sigma}
$$

Consequently, only two images are required to compute the gradient of the surface. The images are first converted into intensity profiles using the column-wise averaging technique described in $[5,6]$. The intensity profiles for the two images are then substituted for $I_{1}(x)$ and $I_{2}(x)$ in Eq. 4 to calculate $p(x)$. A frequency domain integration method described in [17] is used to extract the surface profile from the gradient data. 


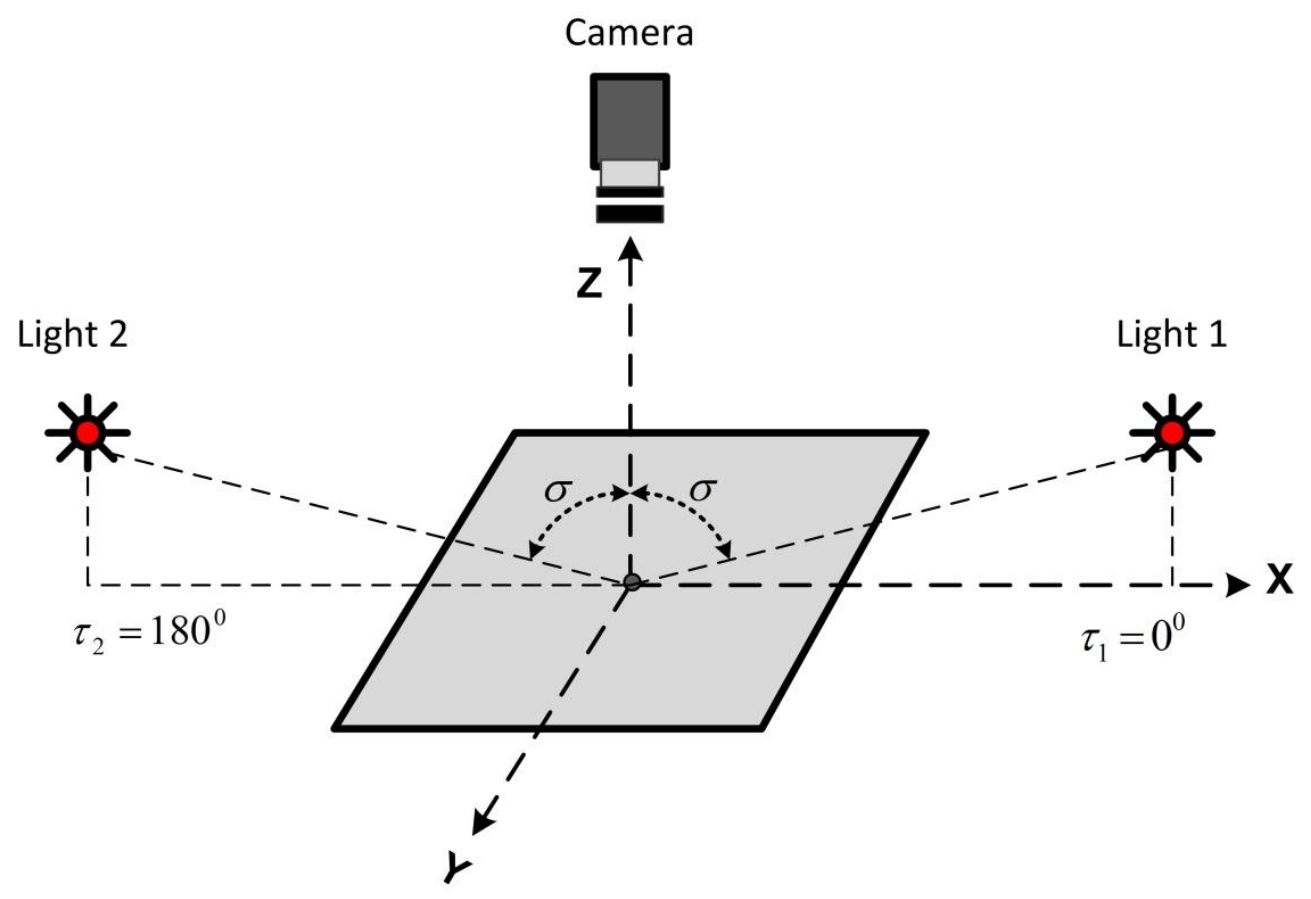

Fig. 2 Schematic of the 2-image photometric stereo setup

\section{Dynamic Photometric Stereo}

The easiest way to marginally overcome the image correspondence problem in dynamic samples has been to capture the image pairs in quick succession, within a very short acquisition time frame. The method is known as temporal multiplexing [7]. In this method, each light source is projected on to the scene at different times, and the image capture is synchronised with the activation of each light in sequence. The drawback of the conventional temporal multiplexing method is that it is only applicable to very slow moving objects. The temporal multiplexing method is adopted in this research but a suitable method is used to match the images before applying the PS technique. The method proposed in this paper is to determine the pixel correspondence between the successive image pairs through the use of an incremental encoder. This makes it suitable for fast moving samples. The pixel displacement between successive image pairs is calculated as follows: 
Suppose that the first and second images are triggered when the surface is at position $p_{1}$ and $p_{2}$ respectively, the linear displacement between image pairs is given as

$$
p=p_{2}-p_{1}
$$

The displacement in pixels can then be calculated as

$$
d_{\text {pixel }}=S R \times p
$$

where $S R$ is the spatial resolution of the image in pixel per millimetre and $p$ is the linear displacement of the surface in millimetres. A sensor is required to measure the position of the workpiece at the time when the acquisition of each image is triggered.

\section{$4 \quad$ Experimental Work}

The success of most machine vision techniques depends on the setup of the light sources. In theory, the PS technique requires that the illumination sources should be distant point light sources, so that uniform illumination across the surface area can be obtained [15]. This requirement cannot be met in practice due to space restrictions and the desire for compact designs. Therefore, Collimated light emitting diodes (LEDs) are used so that this requirement could be at least met. Although rays from collimated lights spread out gradually as they propagate, the divergence is usually minimal. 


\subsection{Optimum Light Source Positions}

The optimum zenith angle is found to be within the range of $80^{\circ}-85^{\circ}$. If the angle of incidence is too close to $90^{\circ}$, the illumination will produce shadowy surfaces. Meanwhile, Lambert's law does not apply to shadowy surfaces. Small angles of incidence $\left(0^{\circ}-30^{\circ}\right)$ also reduce the contrast of the images, thereby reducing the amount of cuttermarks' information that can be revealed [6]. Generally, the only requirement for the locations of the light sources in terms of the azimuth angle is that the lights should not be coplanar. However, it has been observed that only the projections where the light rays are directly perpendicular to the cuttermarks $\left(0^{\circ}\right.$ and $\left.180^{\circ}\right)$ contribute greatly to the performance of the PS method.

Images of a wood surface, captured with light sources positioned at azimuth angles of $0^{\circ}$ and $90^{\circ}$ are shown in Fig. 3. The cuttermarks' ridges are more easily picked up in Fig. 3a compared to Fig. 3b. This indicates that the farther the light sources are positioned away from $90^{\circ}$ or $270^{\circ}$, the better the illumination. Hence, the lights are positioned at angles $\tau_{1}=0^{\circ}$ and $\tau_{2}=180^{\circ}$ as shown in Fig. 2 . 


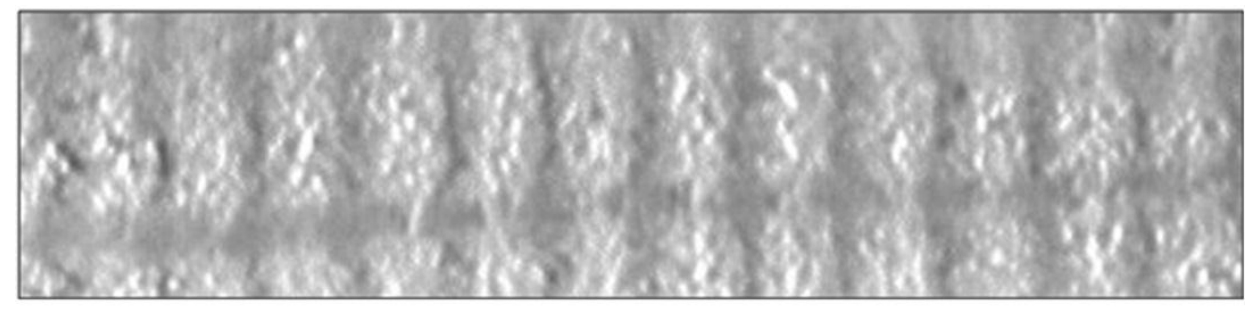

(a) Illuminated from azimuth angle of $0^{\circ}$

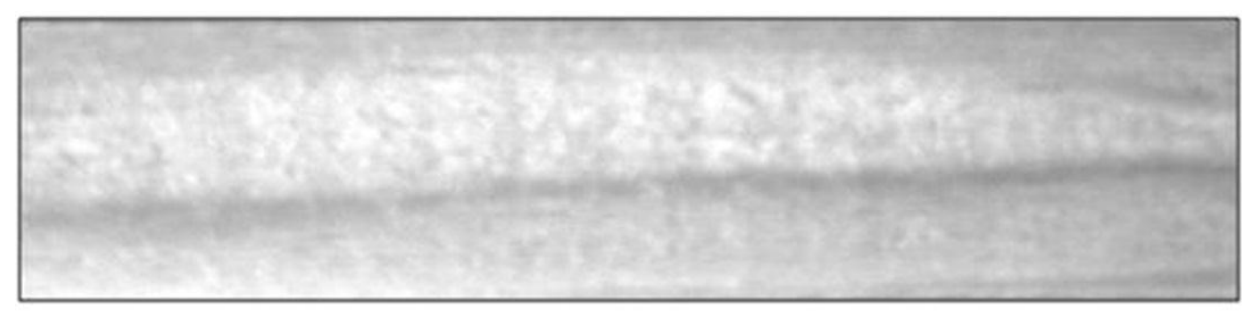

(b) Illuminated from azimuth angle of $90^{\circ}$

Fig. 3 Images of wood obtained from different lightening directions

\subsection{Camera Calibration}

Camera lenses are not perfectly manufactured in practice. The shape of the lenses can lead to radial (barrel or pincushion) distortions of the pixel locations in the images. The effect of this distortion is that a perfectly straight line in the object coordinate appears bent in the images. Tangential distortions can also arise due to the lenses being imperfectly aligned with the image plane during the assembling process [18].

Significant lens distortions will definitely affect the results of the column-by-column averaging of the image intensity data. It is very critical that the variation of the cuttermarks across the feed direction should be maintained in the images. Otherwise the averaging algorithm cannot be used to accurately convert the 3-D profile to a 2-D profile. In order to eliminate the effect of lens distortions, the images have to be corrected before applying the averaging technique. The radial distortions are corrected using a reduced version of Brown's model [19] as given by the following equations: 


$$
\begin{aligned}
& x_{u}=x_{d}+\bar{x} k_{1} r^{2} \\
& y_{u}=y_{d}+\bar{y} k_{1} r^{2}
\end{aligned}
$$

in which

$$
\begin{aligned}
& \bar{x}=x_{d}-x_{o}, \quad \bar{y}=y_{d}-y_{o} \\
& r=\sqrt{\left[\left(x_{d}-x_{o}\right)^{2}+\left(y_{d}-y_{o}\right)^{2}\right]}
\end{aligned}
$$

where

- $\left(x_{d}, y_{d}\right)$ is the original location of the distorted point

- $\left(x_{u}, y_{u}\right)$ is the new location of the corrected point

- $\left(x_{o}, y_{o}\right)$ is the principal point of the image. This is assumed to be located at the centre of the image

- $\mathrm{k}_{1}$ is the coefficient of the $2^{\text {nd }}$ order radial distortion.

It should be noted that the tangential component of distortion has been justifiably neglected to arrive at the above equation because most currently manufactured lenses do not have imperfection in centring. Also, only the second order radial distortion component is considered as recommended for expensive lenses or for lenses with narrow field of view (FOV) [20].

A camera calibration procedure is required to estimate the intrinsic parameter, $\mathrm{k}_{1}$. The method used in this research for the calibration is to target the camera on a 9-by-10 checkerboard pattern of alternating black and with squares. The size of each square after was $2.65 \mathrm{~mm}$ by $2.65 \mathrm{~mm}$. Such a small cell size has been chosen because the FOV of 
the camera is just $52 \mathrm{~mm}$ by $40 \mathrm{~mm}$. The idea is to compute the intrinsic parameters of the camera by acquiring multiple images (30 images) of the calibration pattern under different orientations.

MATLAB camera calibration toolbox developed by the Computational Vision Group in California Institute of Technology was used for the calibration. The toolbox is based on the calibration procedure developed in [21]. It has several built-in functions for computing the camera intrinsic parameters from the acquired multiple images of the known checkerboard pattern. The computed $2^{\text {nd }}$ order radial distortion coefficient is then used to correct for the effects of distortion according to Eq. 7.

\subsection{Test Rig Setup}

A variable-speed conveyor system has been constructed to simulate a real machining environment for the in-process wood surface assessments (Fig. 5). The test rig is capable of measuring moving samples up to the speed of $200 \mathrm{~mm} / \mathrm{s}$. The conveyor belt is equipped with a rotary encoder to measure the distance over which the sample has moved between successive image shots. A 659 x 493 pixel digital camera with an IEEE 1394 FireWire interface is connected to a PC so as to capture the images of the moving surface in real-time. The camera has been carefully aligned perpendicularly to the conveyor belt surface. The illumination sources are red collimated LEDs.

A proximity sensor is used to detect the moving sample when it is within the camera view. The camera is then hardware triggered with each of the two lights on and the other light off. The PC is setup to wait for the two image frames from the camera via the 
FireWire interface using MATLAB Image Acquisition Toolbox. The corresponding positions of the belt (in counts) when the image capture are triggered are also recorded and transmitted to the PC through RS232 serial communication protocol. This tight synchronisation, including the camera triggering, is achieved using a PIC18F458 microcontroller. Since the camera triggering is hardware based, the image acquisition is immediate and the camera latency is minimised.

The displacement between the image frames is calculated according to Eq. 6 in order to establish pixel correspondence. The conversion factor from counts to pixel has been estimated through experimentation (0.2425pixel/count). A small black cross was printed on a white paper and then pasted on a wood sample. The displacements of the cross centre between successive image frames in pixels and encoder counts were then used to determine the conversion factor. 


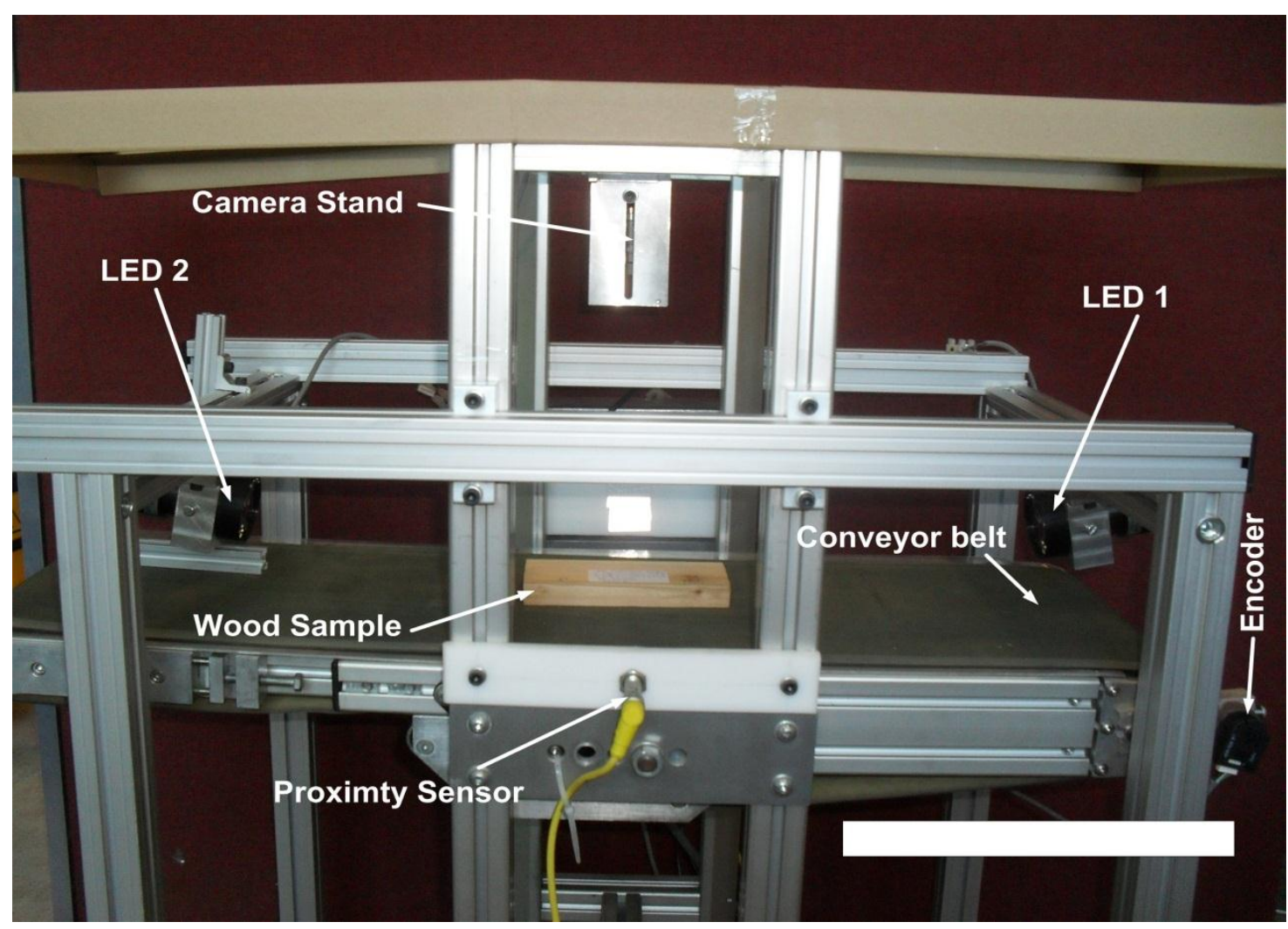

Fig. 4 Dynamic photometric stereo test rig

\subsection{Challenges with the DPS}

There are a number of difficulties that are potentially associated with the application of the proposed technique to moving samples. The major ones being skewing and motion blur within the image frames. Commercial cameras have two types of shutters namely, rolling shutters and global shutters. In the rolling shutter, portions of the objects are exposed to light at different moments in time by scanning horizontally or vertically across the frame. If the wood sample is moving in the horizontal direction during the exposure and the shutter is rolling in the vertical direction, the cuttermarks might become skewed in the final image. Similarly to the radial distortion problem introduced by camera lenses, the averaging of the 3-D profile in order to generate a $2-\mathrm{D}$ profile will 
not give the correct results if the skewness is significant. In that sense, a camera with a global shutter is a better choice for the cuttermarks' measurement.

As opposed to the rolling shutter, the global shutter exposes the entire frame to light at the same time. It eliminates the problem of image skewness, but any motion of the sample during the frame capture would now result into image blurring. Blur will occur if the exposure time is long enough so that the sample can move during one shutter cycle. The problem of motion blur has placed a major constraint on the speed at which the wood sample can be measured. The problem can be somewhat minimised by using a short exposure time (increase shutter speed) as any motion will be frozen in time, resulting in images with less blur. However, enough light might not reach the image sensor if the exposure time is too short, thereby causing the images to be dark. Blurred images could potentially be restored by applying Wiener deconvolution [22].

\subsection{Results and Discussions}

Some wood samples were measured using the test rig. In order to assess the accuracy of the dynamic surface profile measurement, results obtained are compared with those of the static PS. The static PS measurement is used as the baseline reference since its effectiveness has been established in $[5,6]$ by comparing the surface profiles obtained to those from a laser profilometer. The comparisons between the static and the dynamic profiles are shown in Fig. 5 and Fig. 6 for Beech and Oak samples respectively. The vision-based system does not provide absolute height information so the heights have been normalised. 


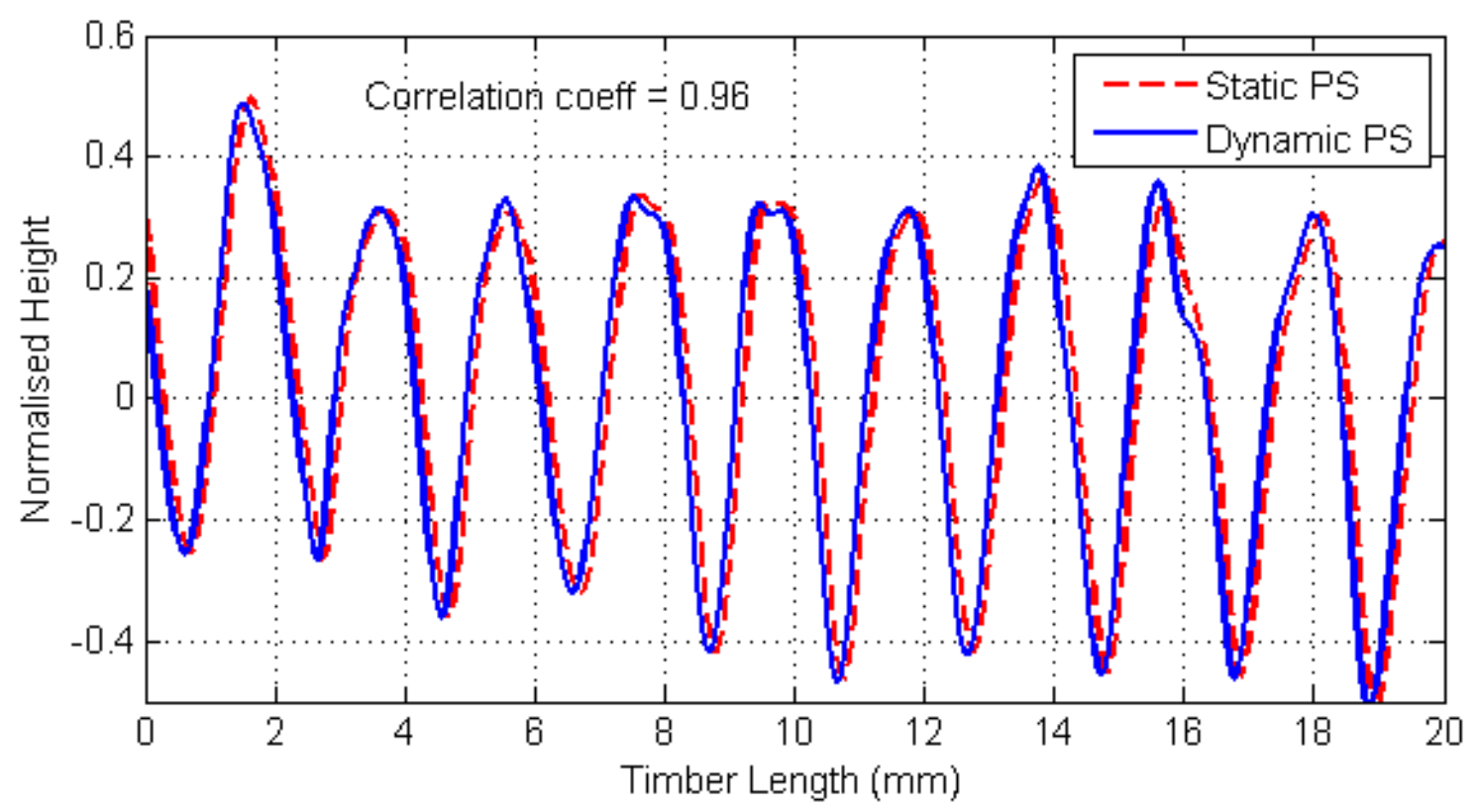

Fig. 5 Static PS profile vs. Dynamic PS profile for Beech sample

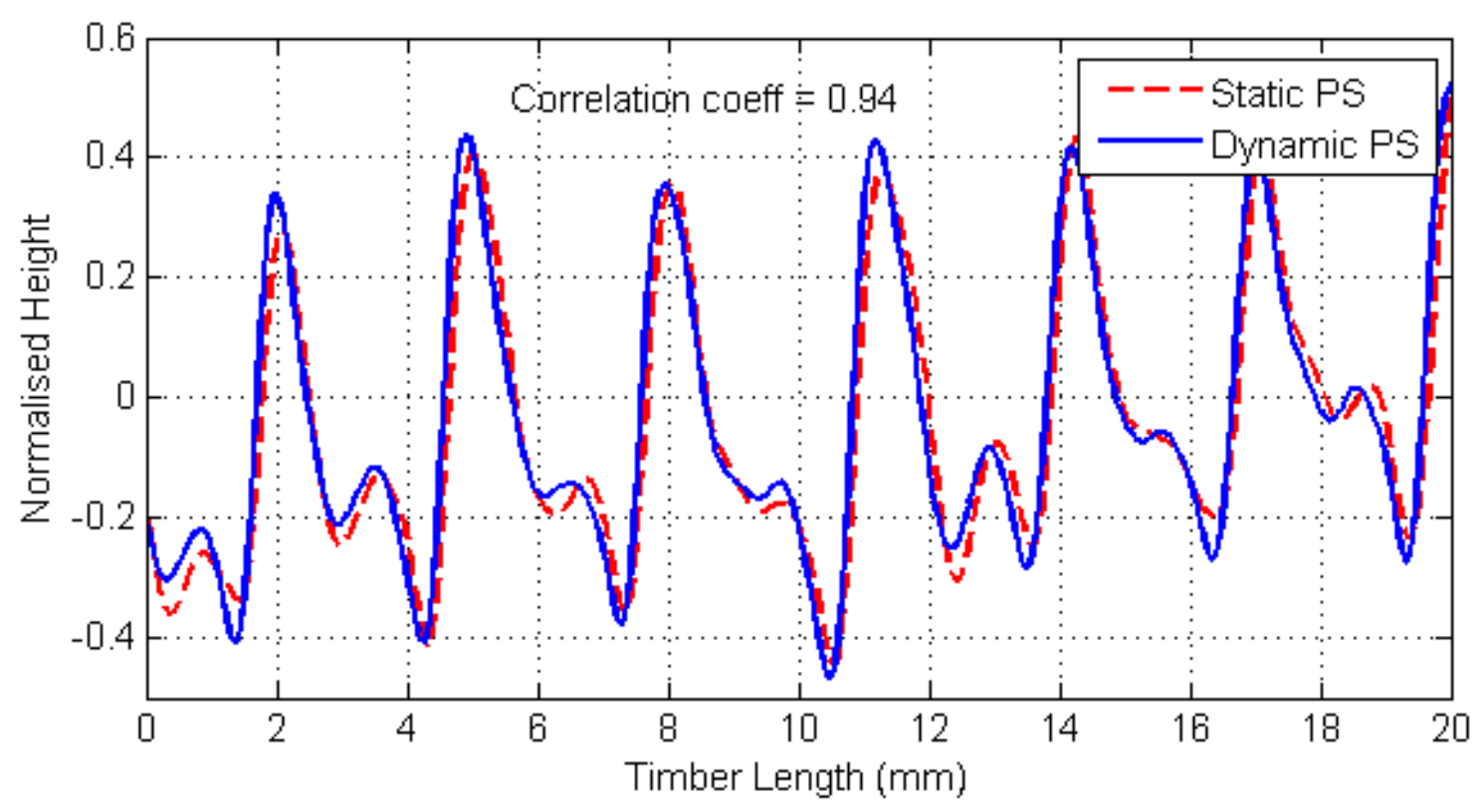

Fig. 6 Static PS profile vs. Dynamic PS profile for Oak sample

The wood samples were machined using the smart planing machine shown in Fig. 7. The machine, which is instrumented with piezoelectric actuators, eddy current sensor and an encoder, is specially designed in order to carry out preliminary investigations 
into alternative ways of improving the performance of industrial wood planing machines. The longer-term aim is to integrate the dynamic surface profile measurement system with the wood planing machine.

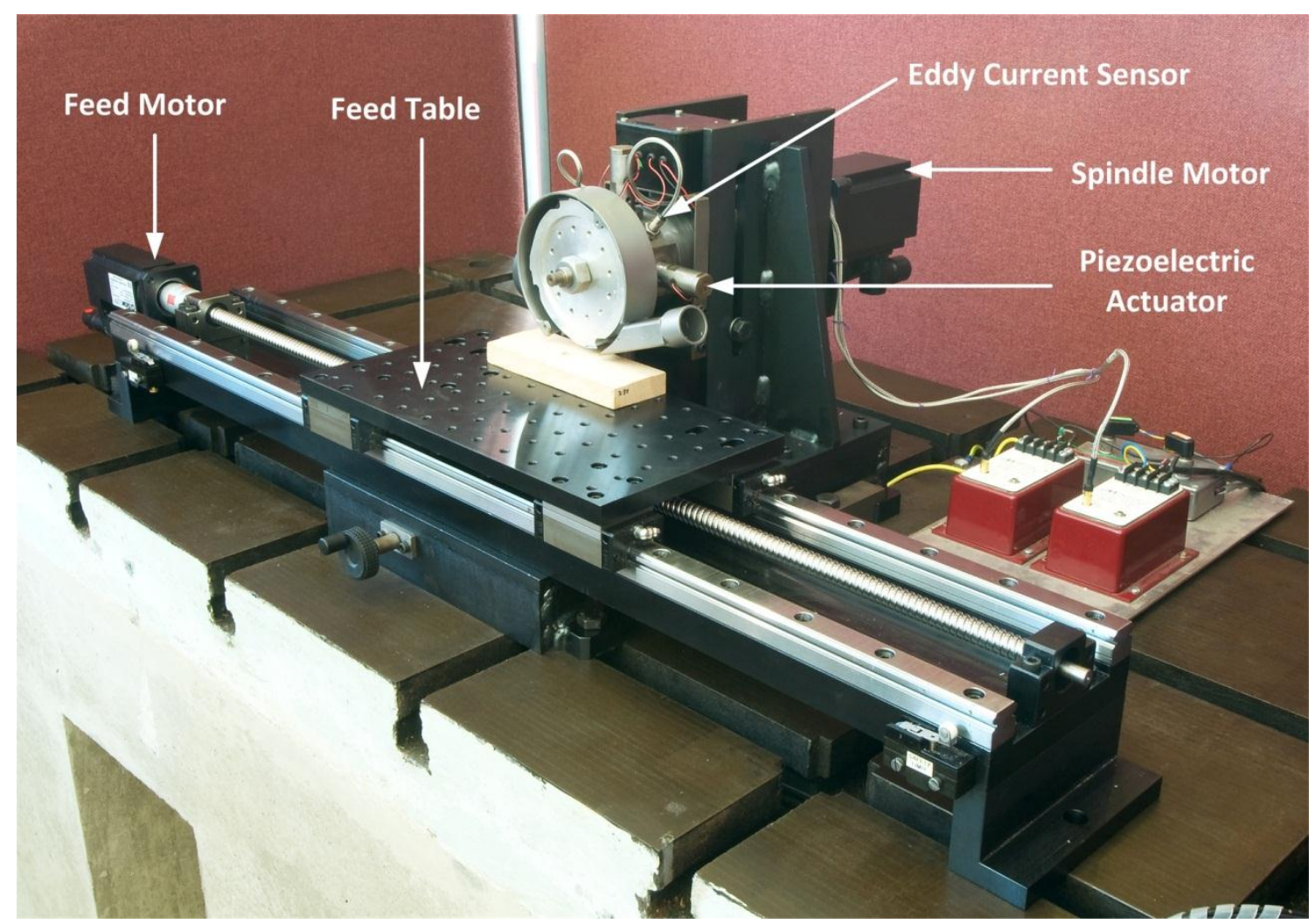

Fig. 7 The smart wood planing machine

The dynamic surface measurement is done for wood samples moving at the speed of $50 \mathrm{~mm} / \mathrm{s}$. Measurements at faster speeds could not be achieved on the current test rig because of motion blur and jerky movement of the conveyor belt. Although the problem of motion blur can be minimised by using higher quality camera and better illumination. The correlation coefficients between the surface profiles obtained using the static and the dynamic PS techniques are given in Table 1. The results show that the dynamic PS 
surface profiles compare well with those of the static PS. The coefficients were computed using the corr2 function in MATLAB according to the following equation:

$$
r=\frac{\sum_{m} \sum_{n}\left(A_{m n}-\bar{A}\right)\left(B_{m n}-\bar{B}\right)}{\sqrt{\left(\sum_{m} \sum_{n}\left(A_{m n}-\bar{A}\right)^{2}\right)\left(\left(\sum_{m} \sum_{n}\left(B_{m n}-\bar{B}\right)^{2}\right)\right)}}
$$

where

- $\quad A$ and $B$ are vectors representing the static and dynamic profiles respectively

- $\bar{A}$ and $\bar{B}$ are the mean values of the elements in $A$ and $B$ respectively

Table $1 \quad$ Percentage correlation between static and dynamic surface profile measurements for some selected wood samples

\begin{tabular}{|c|c|c|c|}
\hline Sample & Wood Species & $\begin{array}{c}\text { Cuttermarks } \\
\text { Width }\end{array}$ & $\begin{array}{c}\text { Correlation } \\
\text { Coefficient }\end{array}$ \\
\hline 1 & Beech & $2 \mathrm{~mm}$ & $96 \%$ \\
\hline 2 & Oak & $2 \mathrm{~mm}$ & $94 \%$ \\
\hline 3 & Ramin & $2.5 \mathrm{~mm}$ & $93 \%$ \\
\hline 4 & Spruce & $1.5 \mathrm{~mm}$ & $87 \%$ \\
\hline 5 & Beech & $2.5 \mathrm{~mm}$ & $96 \%$ \\
\hline 6 & Meranti & $2.5 \mathrm{~mm}$ & $91 \%$ \\
\hline
\end{tabular}

Apart from motion blur and image skewness, the conveyor belt slippage is another potential source of errors in matching the photometric image pair. Under this situation, the actual wood sample movement will be different from the rotary sensor readings. The belt slippage problem will not occur when the vision system is finally integrated with the wood planing machine since the feed table is being driven by a lead screw. 
Considering the fact that any matching method employed will be subject to some errors, it is necessary to investigate the robustness of the PS technique to marginal image mismatches. This is done by comparing the surface profile extracted from perfectly matched images with the profiles extracted from manually mismatched images. Comparisons between the profiles obtained from the perfectly matched images and those of 8 and 15 pixels mismatched images are shown in Fig. 8 and Fig. 9 respectively.

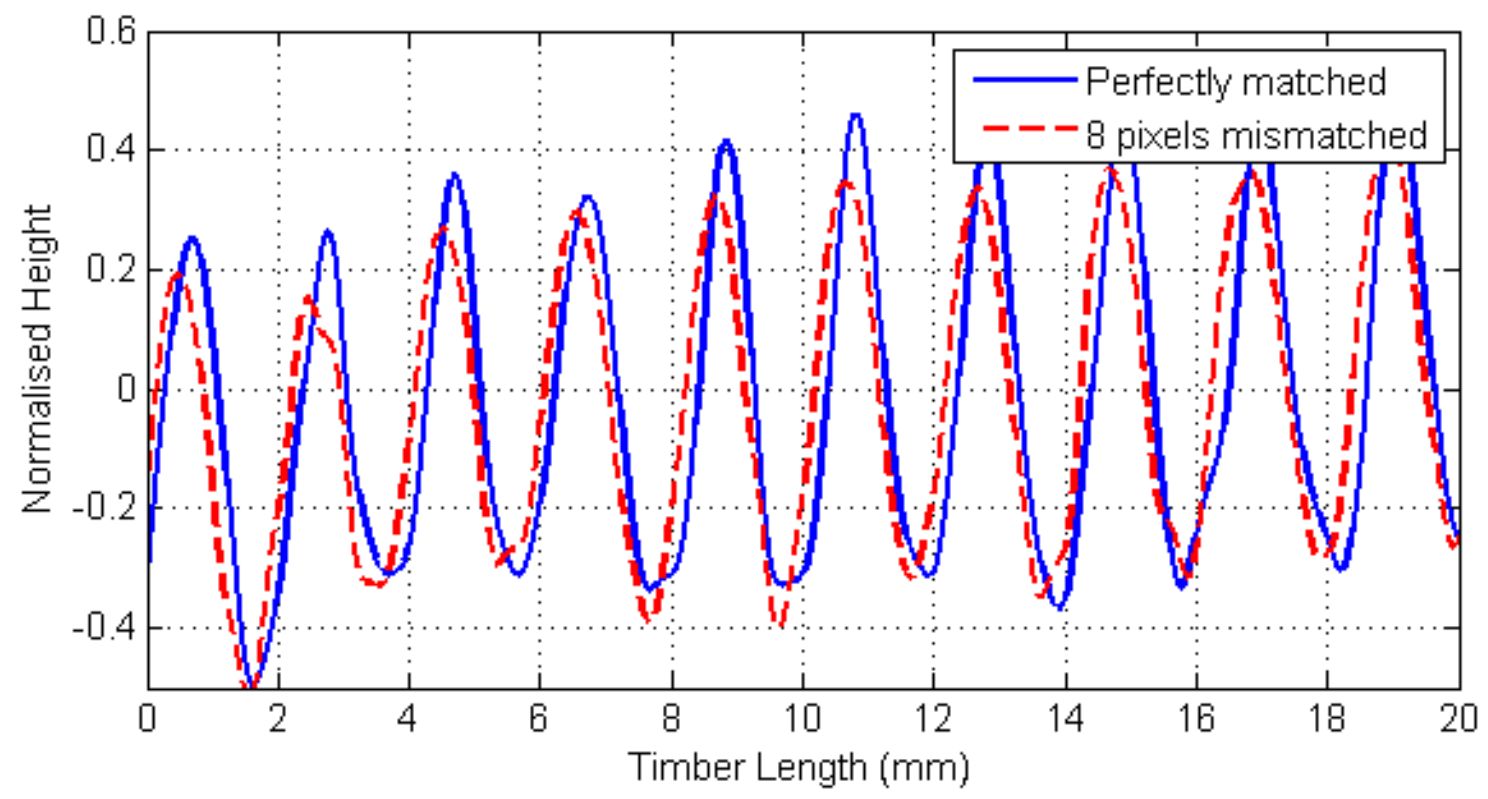

Fig. 8 Comparisons between perfectly matched and 8 pixels mismatched profile 


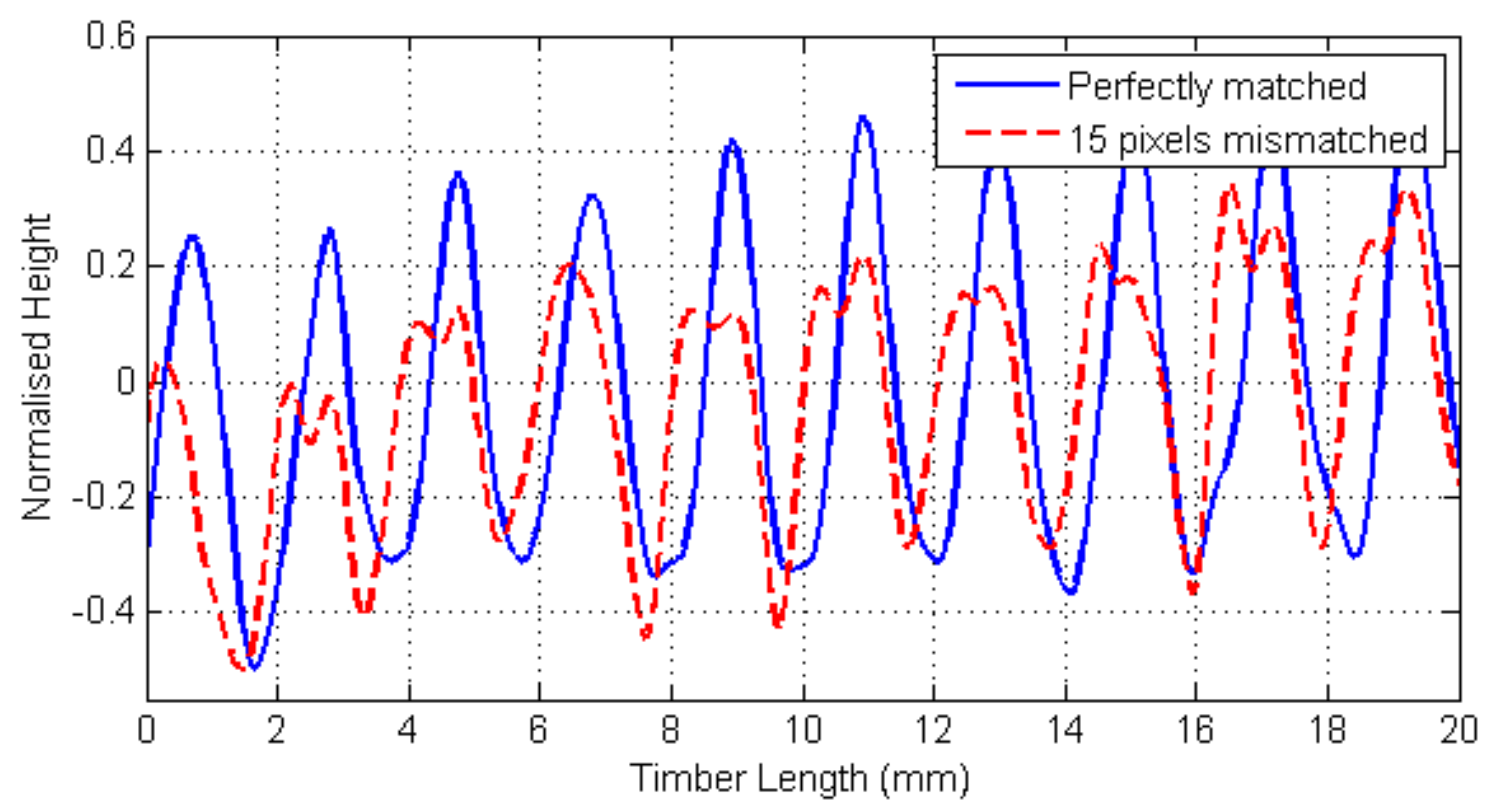

Fig. 9 Comparisons between perfectly matched and 15 pixels mismatched profile

The correlation between the perfectly matched surface profile and the eight pixels mismatched profile is about $87 \%$. There is only about $56 \%$ correlation between the perfectly matched profile and the fifteen pixels mismatched profile. Based on these values, a mismatch up to about eight pixels could still produce acceptable results. This means that the dynamic PS approach is robust to minimal matching errors. The tolerance limit will actually depend on the sizes of the cuttermarks and the spatial resolution of the images.

\section{Conclusions}

An adaptation of the PS technique to the assessment of moving wood samples is presented in this paper. The CPS technique has been used earlier to assess surface waviness on static samples. The strict requirement of the CPS is that the view position must remain fixed with respect to the camera during the acquisition of the photometric images. While this requirement is readily satisfied by static samples, it is found to be the 
most restrictive for moving samples. The major challenge in the application of the CPS technique to moving samples is how to determine the pixel correspondence between the photometric image pairs.

A method known as temporal multiplexing is used to control the acquisition of the image pairs under different lightening directions. An encoder is then used to determine the displacements of the sample between successive image pairs in order to establish pixel correspondence. Experiments performed on a test rig designed to simulate a real machining environment show that the approach is suitable for in-process surface quality inspection of moving samples. Future work will be focused on increasing the speed at which the samples can be measured and also utilising the information extracted from the measurements to provide outer loop control of the wood machining process in order to produce higher quality surface finish.

\section{Acknowledgement}

This work was supported by the EPSRC Innovative Manufacturing and Construction Research Centre at Loughborough University, UK.

\section{References}

[1] Jackson, M. R., Parkin, R. M., Brown, N. Waves on wood. Proceedings of the Institution of Mechanical Engineers, Part B: Journal of Engineering Manufacture, 2002, 216 (4), 475-497. 
[2] Elmas, S., Islam, N., Jackson, M. R., Parkin, R. M. Analysis of profile measurement techniques employed to surfaces planed by an active machining system. Measurement, 2011, 44 (2), 365 - 377.

[3] Hesselbach, J., Hoffmeister, H. W., Loohb, T. Process-integrated quality assurance in wood machining centres with the help of image processing. Journal of Production Engineering Research and Development, 2007, 1 (1), 97-101. ISSN 1863-7353.

[4] Yang, D., Jackson, M. R., Parkin, R. M. Inspection of wood surface waviness defects using the light sectioning method. Proceedings of the Institution of Mechanical Engineers, Part I: Journal of Systems Control and Engineering, 2006, 220 (7), 617-626.

[5] Jackson, M., Yang, D., Parkin, R. Analysis of wood surface waviness with a two-image photometric stereo method. Proceedings of the Institution of Mechanical Engineers, Part I: Journal of Systems and Control Engineering, 2007, 222, 1091-1099.

[6] Yang, D. Measurement of cutter marks on planed wood surfaces with machine vision methods. PhD Thesis, Loughborough University, 2006. 
[7] Smith, M. L., Smith, L. N. Dynamic photometric stereo - a new technique for moving surface analysis. Image and Vision Computing, 2005, 23 (9), 841-852.

[8] Farooq, A. R., Smith, M. L., Smith, L. N., Midha, S. Dynamic photometric stereo for online quality control of ceramic tiles. Computers In Industry, 2005, $56,918-934$.

[9] Farooq, A. R. Dynamic photometric stereo for complex surface analysis. Phd Thesis, University of the West England, Bristol, 2004.

[10] Bringier, B., Helbert, D., Khoudeir, M. The photometric reconstruction of a dynamic textured surface from just one color image acquisition. Journal of the Optical Society of America, 2008, 25 (3), 566 - 574.

[11] Smith M. L., Smith, G., Hill, T. Gradient space analysis of surface defects using photometric stereo derived bump map. Image and Vision Computing, 1999, 17 (3-4), 321-332.

[12] Smith, M. L. The analysis of surface texture using photometric stereo acquisition and gradient space domain mapping. Image and Vision Computing, 1999, 17 (14), 1009-1019. 
[13] Tagare, H. D., Defigueiredo, R. J. Simultaneous estimation of shape and reflectance map from photometric stereo. CVGIP: Image Understanding, 1992, 55 (3), 275-286.

[14] Maristany, A. G., Lebow, P. K., Brunner, C. C., Butler, D. A., Funck, J. W. Classifying wood surface features using dichromatic reflection. In Proceedings of the SPIE - The International Society for Optical Engineering, 1992, 1836, 56 -64 .

[15] Jain, R. C., Kasturi, R., Schunk, B. G. Machine vision. New York: McGraw-Hill Inc; 1995, ISBN 0-07-113407-7.

[16] Smith, M. L. Surface inspection techniques using the integration of innovative machine vision and graphical modelling techniques. London and Bury St Edmunds: Professional Engineering Publishing Limited; 2001, ISBN 186058 2923.

[17] Frankot, R. T., Chellappa, R. A method for enforcing integrability in shape from shading algorithms. IEEE Transactions on Pattern Analysis and Machine Intelligence, 1988, 10 (4), 439-451.

[18] Bradski G., Kaehler A. Learning OpenCV: Computer vision with the OpenCV library. Sebastopol CA: O’really Media Inc.; 2008, ISBN 978-0-596-51613-0. 
[19] Brown, D. C. Close-range camera calibration. Photogrammetric Engineering, $1971,37(8), 855-866$.

[20] Zhang, Z. Flexible camera calibration by viewing a plane from unknown orientations. The Proceedings of the $7^{\text {th }}$ IEEE International Conference on Computer Vision, Sept 20-27, 1999, Kerkyra, Greece, pp 666- 673, ISBN 07695-0164-8.

[21] Heikkila, J., Silvan, O. A four-step camera calibration procedure with implicit image correction. Proceedings of the 1997 IEEE Computer Society Conference on Computer Vision and Pattern Recognition, June 17-19, 1997, San Juan, Puerto Rico, pp. 1106 - 1112, ISSN 0-8186-7822-4.

[22] Wu, Q., Merchant, F. A., Castleman, K. R. Microscope image processing. London: Academic Press; 2008, ISBN 978-0123725783. 